\title{
Enhancement of delay eyelid conditioning by microcurrent electrical stimulation of the medial prefrontal cortex is triggered by the expression of Fos protein in guinea pigs
}

\author{
YA-JUAN ZHENG, YU-CHEN DONG, CHAO ZHU and MEI-SHENG ZHAO \\ Department of Ophthalmology, Eye Hospital of the Second Clinical Hospital \\ of Jilin University, Changchun, Jilin 130051, P.R. China
}

Received February 8, 2014; Accepted June 19, 2014

DOI: $10.3892 /$ etm.2016.2987

\begin{abstract}
Eyelid conditioning, including delay eyelid conditioning and trace eyelid conditioning, has been used extensively to study neural structures and mechanisms of learning and memory as a form of associative learning. In the present study, microcurrent electrical stimulation was used to stimulate the medial prefrontal cortex (mPFC) to induce delay eyelid conditioning in guinea pigs. The acquisition rate and relative latency of the conditioned eyelid response (CR) and the startle eyelid response (SR) were analyzed. The $\mathrm{mPFC}$ sites in the guinea pigs were examined under a light microscope following Nissl staining. In addition, the expression of Fos protein in neurons was detected using immunohistochemistry and western blot analysis. The results indicated that the acquisition rates of $\mathrm{CR}$ and SR were increased significantly $(\mathrm{P}<0.05)$, whilst the relative latencies of $\mathrm{CR}$ and $\mathrm{SR}$ were decreased significantly $(\mathrm{P}<0.05)$. Lesions were observed in the mPFC regions in the training group when compared with the control group. In addition, immunohistochemistry and western blot analysis revealed that Fos expression was significantly increased in the training group when compared with the sham group for the control and resident-intruder test guinea pigs $(\mathrm{P}<0.05)$. Therefore, the enhancement of delay eyelid conditioning by microcurrent electrical stimulation of the $\mathrm{mPFC}$ in guinea pigs was triggered by the expression of Fos protein. The observations of the present study further expand the understanding of conditioned reflexes and may aid future investigations into the formation of eyelid conditioning and the mechanisms underlying the circuit in various conditions.
\end{abstract}

Correspondence to: Professor Mei-Sheng Zhao, Department of Ophthalmology, Eye Hospital of the Second Clinical Hospital of Jilin University, 218 Zhiqiang Road, Nan'guan, Changchun, Jilin 130051, P.R. China

E-mail: zhaomeishengdr@yeah.net

Key words: delay eyelid conditioning, medial prefrontal cortex, Fos protein, guinea pigs

\section{Introduction}

Pavlovian eyelid conditioning is an important method for tracing the pathway of the projection of fibers, examining the acquisition or retention of memory and verifying the process of learning $(1,2)$. A conditioned response (CR) may be established by pairing a conditioned stimulus (CS), which does not elicit the eyelid reflex spontaneously, with an unconditioned stimulus (US) that naturally elicits the eyelid reflex (3). To date, studies on eyelid conditioning using lesions, inactivation, stimulation and neural tract tracing have provided information on the pathways in the cerebellum, cerebrum and brain stem.

Usually, peripheral stimuli are used as a CS; for example, light CS to the eye, or a tone CS to the ear. Alternatively, somatic stimuli may be used to establish a CR. Further investigations were performed by directly stimulating the central nervous system. Knowlton et al (4) used an auditory stimulus to establish an eyelid CR. An eyelid CR was also observed in the experiment performed by Green et al (5), where the CS and US were transduced directly into the interpositus nucleus (5). The results from the study by Knowlton and Thompson (6) demonstrated that the average length of time to establish eyelid conditioning by a central CS was shorter compared with a peripheral CS (6). In addition, a number of previous studies $(5,6)$ have demonstrated that central CS can establish eyelid conditioning faster and more effectively compared with peripheral CS.

Central CS differ from peripheral CS, as a central CS does not stimulate the peripheral receptor directly, but affects central areas through which peripheral messages are transduced into the central nervous system, such as lateral pons (7). Other CS stimulate offsets of projections, including the projection pathway in the cerebellum and parallel fibers.

However, whether stimulating the transduction terminal in the cerebral cortex is able to establish eyelid conditioning and influence the rate of acquisition is yet to be elucidated. Furthermore, differences between the effects of delay eyelid conditioning and trace eyelid conditioning are yet to be determined, as well as the underlying mechanisms. Investigating these areas is important to establish mechanisms of CS. Although a number of associations have been demonstrated between the medial prefrontal cortex (mPFC) and delay 
eyelid conditioning $(8,9)$, the present study, for the first time, investigated the effects of microcurrent electrical stimulation of the mPFC on the induction of delay eyelid conditioning in guinea pigs.

\section{Materials and methods}

Animals. In total, eight male guinea pigs (weight, 330-430 g; Animal Center of Jilin University, Changchun, China) were used in this study. The animals were housed in standard plastic cages and maintained on a 12-h light/dark cycle with light onset at 7:00 a.m. Food and water were provided ad libitum and the temperature was maintained at $25 \pm 1^{\circ} \mathrm{C}$. All experiments were performed between 8:00 a.m. and 6:00 p.m., during the light portion of the cycle. Experimental procedures were approved by the Animal Care Committee of Jilin University (Changchun, China) and were in accordance with the principles outlined in the National Institutes of Health Guide for the Care and Use of Laboratory Animals (Bethesda, MD, USA).

Surgery. The guinea pigs were allowed to acclimatize to the cages for one week prior to surgery. The animals were anesthetized with an intraperitoneal (i.p.) injection of a mixture of ketamine $(0.3 \mathrm{ml} / \mathrm{kg}$; Jiangsu Hengrui Medicine Co., Ltd., Lianyungang, China) and sumianxin II $(0.05 \mathrm{ml} / \mathrm{kg}$; Veterinarian Institute of Military Medical Science Academy, Beijing, China). The head of the anesthetized animal was secured to a stereotaxic apparatus (SR-6N; Narishige International, Ltd., Tokyo, Japan) with the lambda positioned $1.0 \mathrm{~mm}$ ventral to the bregma. Next, a stimulating electrode was implanted and the coordinates obtained from the bregma were $2.0 \mathrm{~mm}$ posterior and $0.7 \mathrm{~mm}$ lateral. Four stainless steel screws connected by one conductor were attached to the skull as a reference electrode. A small metal probe (Tiangen, Beijing, China), which was used to attach the left upper eyelid to a movement-measuring device, was sutured into, but not through, the edge of the left upper eyelid. Following surgery, the animals were injected with gentamycin sulfate $(5 \mathrm{mg} / \mathrm{kg}$ i.p.; North China Pharmaceutical Corporation, Shijiazhuang, China) and benzylpenicillin sodium (10 mg/kg i.p.; North China Pharmaceutical Corporation) every $12 \mathrm{~h}$ for five days and allowed one week of recovery. In addition, the mPFC sites were examined under a light microscope (CX31; Olympus Corp., Tokyo, Japan) following Nissl staining $(6,8)$.

Apparatus. Eyelid movements were measured using a high-resolution spring-return potentiometer (JZJ01; Cheng Yi, Chengdu, China), attached via a thread lead hooked through the nylon loop sutured into the left upper eyelid. A stimulator (YC-2; Chengdu, China), equipped with a constant current and stimulus isolation, was used to deliver an electronic CS, while a plastic pipe placed $1.0 \mathrm{~cm}$ from the left eyeball was used to deliver a corneal air puff (US). The CS and US were controlled using a computer-monitored system. Eyelid movement mechanogram and markers of the applied stimuli were digitized at a sample rate of $20 \mathrm{kHz}$ using a data acquisition system (RM6280C; Cheng Yi) and were acquired using the built-in software (version 4.7; Microsoft Corp., Redmond, WA, USA). A Windows PC was used to store and analyze the behavioral data.
Training. Guinea pigs were allowed to adapt to the experimental environment for two days $(80 \mathrm{~min} / \mathrm{day})$ prior to the training sessions. The daily training sessions included 50 trials of classical delay eyelid conditioning with an interval of 15-30 sec, during which the animals were restrained in a Plexiglas container $(25 \times 15 \times 15 \mathrm{~cm})$ located in a sound- and light-attenuating chamber, and their heads were secured with blunt ear-bars pressing on the head-stages. All 50 trials consisted of paired presentations of CS and US. The CS, electrical stimulation of the mPFC, was administered in a $1,000 \mathrm{~Hz}$ train of $450 \mu \mathrm{A}$ monophasic pulses for $250 \mathrm{msec}$. The US was a mild puff of air to the eyes $(3 \psi)$ for $100 \mathrm{msec}$.

Resident-intruder (RI) test. Three weeks prior to the start of the experiment, a male guinea pig from the training group (mPFC operated group) was housed with a female rat to stimulate territorial behavior. A novel young male intruder guinea pig was exposed to a male resident rat (mPFC male guinea pigs) for $15 \mathrm{~min}$. The female was removed $30 \mathrm{~min}$ prior to the test. Behaviors were recorded using a digital video camera (LS20M; Olympus Corp.). In the present experiments, the intruder guinea pigs were not aggressive and no intruder guinea pigs initiated aggression.

Grouping. The pigs were divided into two groups, including the training (in which medial prefrontal cortex was stimulated by microcurrent electrical treatment) and sham guinea (in which pigs did not receive any treatment) groups. Furthermore, the pigs were divided into the RI test (in which pigs underwent the RI test) and RI control (in which pigs were not subjected to the RI test) groups.

\section{Immunohistochemical detection of Fos protein in the neurons} of guinea pigs. For immunocytochemical detection of Fos protein expression, the guinea pigs were anesthetized with Avertin (Sigma-Aldrich, Carlsbad, CA, USA) $2 \mathrm{~h}$ following the initiation of the RI test and perfused with paraformaldehyde. The sham and training groups of the mPFC-operated guinea pigs were housed alone until perfusion. The brains were processed for immunocytochemical detection of Fos protein expression, as previously described by Wang et al (10).

Western blot analysis. Tissue samples $(20 \mathrm{mg})$ were homogenized in $200 \mu 1$ ice-cold lysis buffer (Sigma-Aldrich), supplemented with phenylmethylsulfonyl fluoride, and centrifuged at $12,000 \mathrm{xg}$ for $10 \mathrm{~min}$ at $4^{\circ} \mathrm{C}$, following which the supernatants were collected. Equal quantities of protein from each sample were subjected to SDS-PAGE (10\% polyacrylamide) and transferred onto polyvinylidene difluoride membranes (Millipore, Bedford, MA, USA). The membranes were blocked for $2 \mathrm{~h}$ with $1 \%$ bovine serum albumin, then incubated overnight at $4^{\circ} \mathrm{C}$ with a mouse monoclonal anti-Fos antibody (1:2,000; sc-52; Santa Cruz Biotechnology, Inc., Santa Cruz, CA, USA). The membranes were washed and incubated with a polyclonal goat anti-mouse IgG secondary antibody conjugated to horseradish peroxidase $(1: 1,000$; sc-395763; Santa Cruz Biotechnology, Inc.). Immunoreactive bands were visualized with a SuperSignal West Pico enhanced chemiluminescence kit (Pierce Biotechnology, Inc., Rockford, IL, USA), according to the manufacturer's instructions. Band 
A

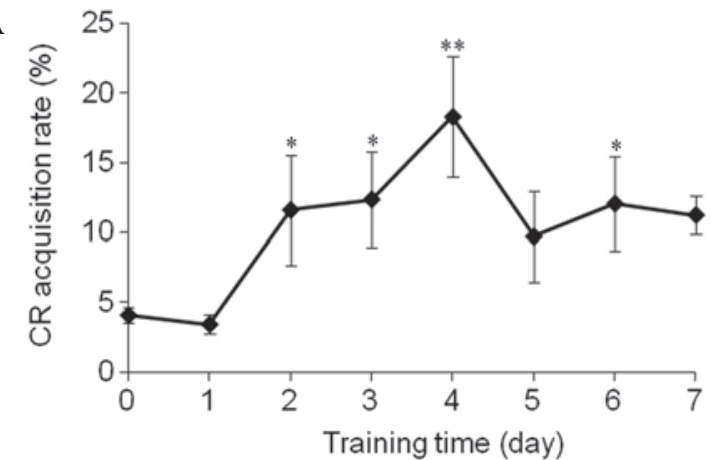

B

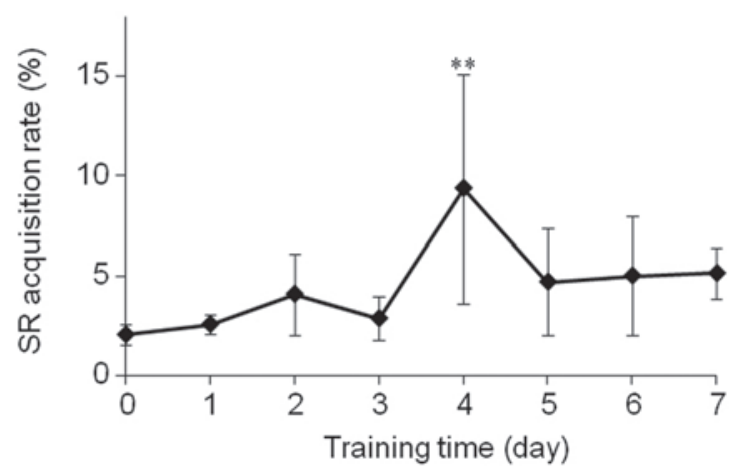

Figure 1. Acquisition rates of the (A) CR and (B) SR in delay eyelid conditioning, developed using microcurrent electrical stimulation of the medial prefrontal cortex. Day 0 indicates no training. Each experiment was repeated seven times and data are expressed as the mean \pm standard error of the mean. ${ }^{*} \mathrm{P}<0.05$ and ${ }^{* *} \mathrm{P}<0.01$, vs. day 0 . CR, conditioned eyelid response; $\mathrm{SR}$, startle eyelid response.

A

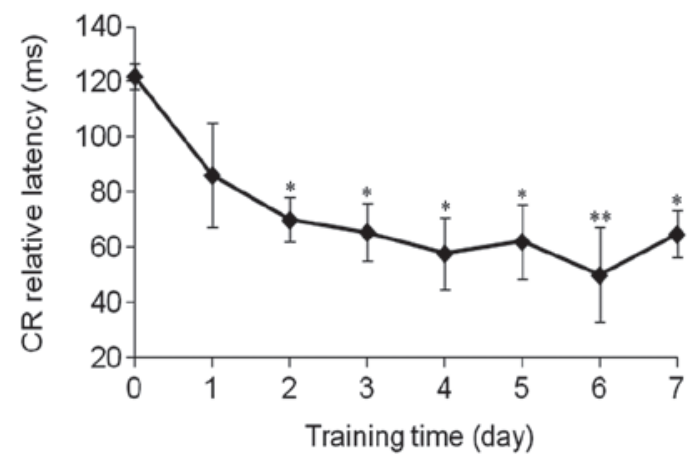

B

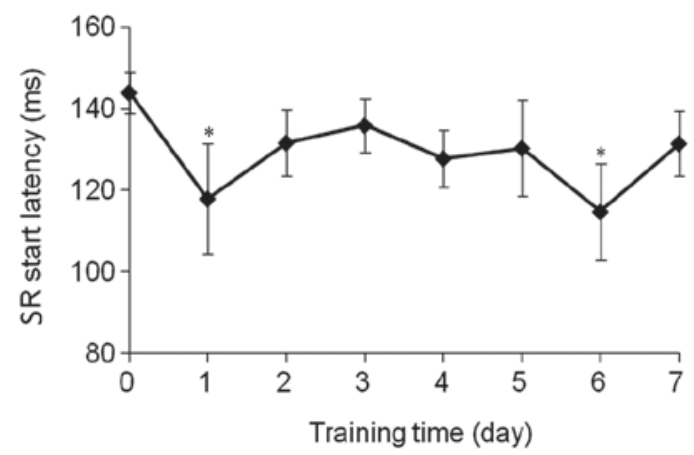

Figure 2. Relative latency of the (A) CR and (B) SR in delay eyelid conditioning, developed using microcurrent electrical stimulation of the medial prefrontal cortex. Day 0 indicates no training. Each experiment was repeated seven times and data are expressed as the mean \pm standard error of the mean. ${ }^{*}<0.05$ and ${ }^{* *} \mathrm{P}<0.01$, vs. day 0 . CR, conditioned eyelid response; $\mathrm{SR}$, startle eyelid response.

intensities were quantified using Quantity One software (Bio-Rad Laboratories, Hercules, CA, USA).

Statistical analysis. All data are expressed as the mean \pm standard error of the mean. Statistical significance was determined using the t-test with SPSS software package version 18.0 (SPSS, Inc., Chicago, IL, USA), where $\mathrm{P}<0.05$ was considered to indicate a statistically significant difference.

\section{Results}

Acquisition rate of $C R$ and SR. After seven days of CS-US paired training, the acquisition rate of the CR exhibited an unstable increase and reached $11.24 \pm 1.40 \%$, which was significantly higher compared with the guinea pigs that had not received training $(\mathrm{P}<0.01$; Fig. 1A). Furthermore, the acquisition rate of the SR also exhibited a slight increase (Fig. 1B).

Relative latency period of the $C R$ and SR. The relative latency period of the CR significantly decreased between $122.0 \pm 4.5$ and $64.8 \pm 8.5 \mathrm{msec}(\mathrm{P}<0.01$; Fig. $2 \mathrm{~A})$, and a decrease in the start latency period of the SR was also observed (Fig. 2B).

Examination of the $\mathrm{MPFC}$ in the training and control guinea pigs. Examination of the MPFC with Nissl staining revealed lesions in the $\mathrm{mPFC}$ regions in the training group when compared with the control group (Fig. 3). This observation indicated that training may trigger changes in the mPFC region of guinea pigs.

Effect of mPFC lesions on the expression levels of Fos in the neurons of the guinea pigs in the training group. To examine the effects of mPFC lesions on the neurons in the training group guinea pigs, the expression levels of Fos protein were investigated in the training and control guinea pigs. As shown in Fig. 3, the expression of Fos protein in the training group guinea pigs was significantly higher compared with the sham group, for the control pigs and RI test guinea pigs $(\mathrm{P}<0.01$; Fig. 4).

Fos protein expression increases following training in guinea pigs. In order to confirm Fos expression in the training group guinea pigs, Fos protein was detected in the neurons using western blot analysis. The results revealed that Fos expression was significantly increased in the training group compared with the sham group $(\mathrm{P}<0.01$; Fig. 5). Furthermore, for the RI test pigs, Fos expression increased in the training group when compared with sham group $(\mathrm{P}<0.01$; Fig. 5$)$.

\section{Discussion}

The understanding of the mechanisms underlying eyelid conditioning, including delay eyelid conditioning and trace eyelid conditioning, has increased significantly in recent decades. 

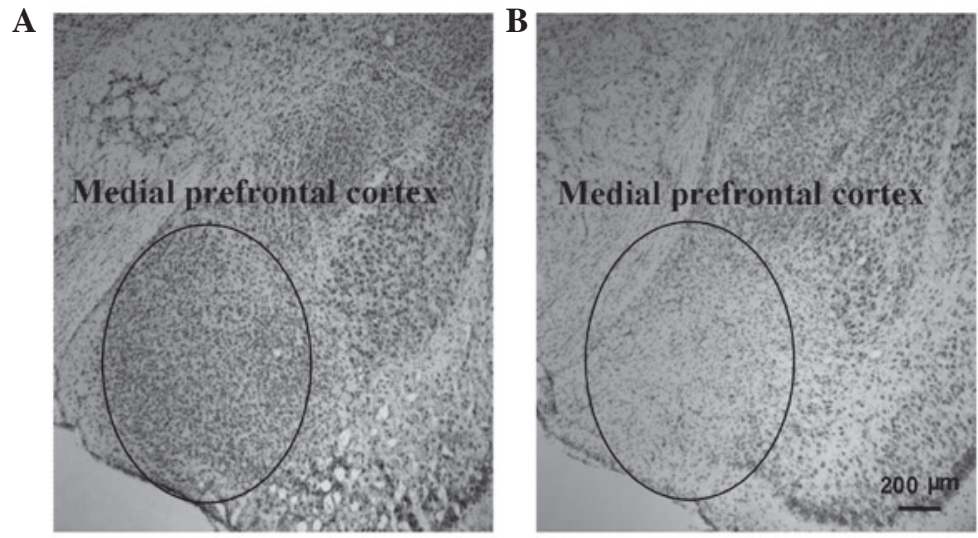

Figure 3. Lesion sites of the medial prefrontal cortex (mPFC) in the (A) training and (B) sham guinea pigs. Lesions were confined within the mPFC; the circles indicate the lesion area (scare bar, $200 \mu \mathrm{m}$ ).
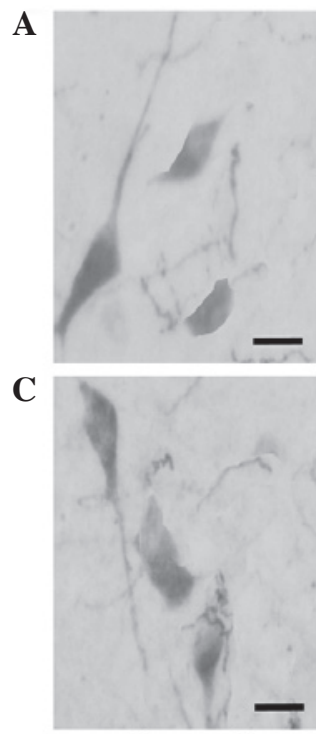

Control

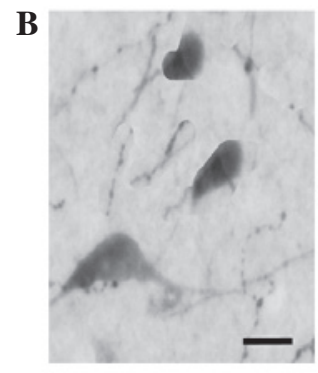

D

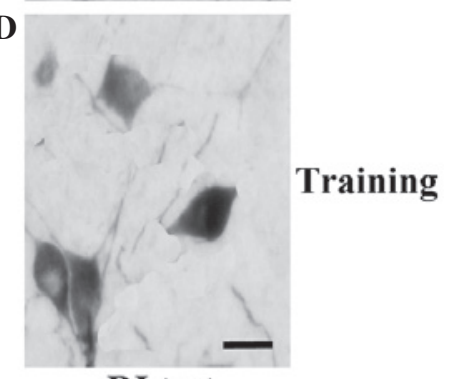

RI test
$\mathbf{E}$

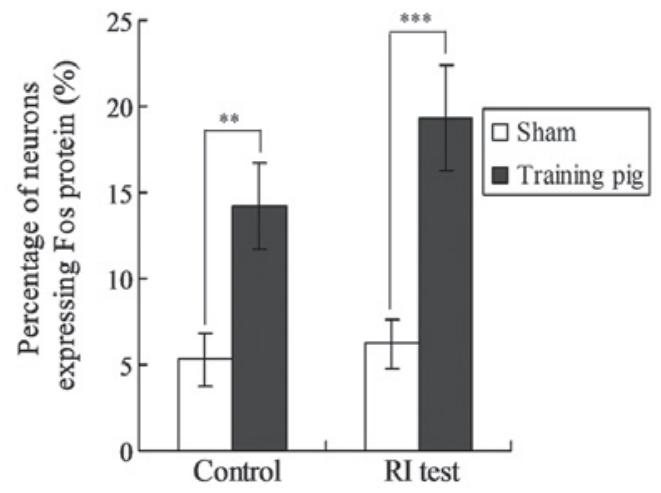

Figure 4. Effects of medial prefrontal cortex lesions on the expression of Fos protein in the neurons of guinea pigs. Expression levels of Fos protein in the neurons of guinea pigs in the (A and B) sham and (C and D) training groups (scale bars, $50 \mu \mathrm{m}$ ). (E) Relative expression levels with statistical analysis. ${ }^{* *} \mathrm{P}<0.01$ and ${ }^{* * *} \mathrm{P}<0.001$, vs. control guinea pigs. RI, resident-intruder.

Eyelid conditioning is primarily associated with cerebellar interpositus nuclei and brainstem nuclei $(11,12)$; however, it has also been shown to be involved with the mPFC $(13,14)$. Therefore, in the present study, CS was performed on
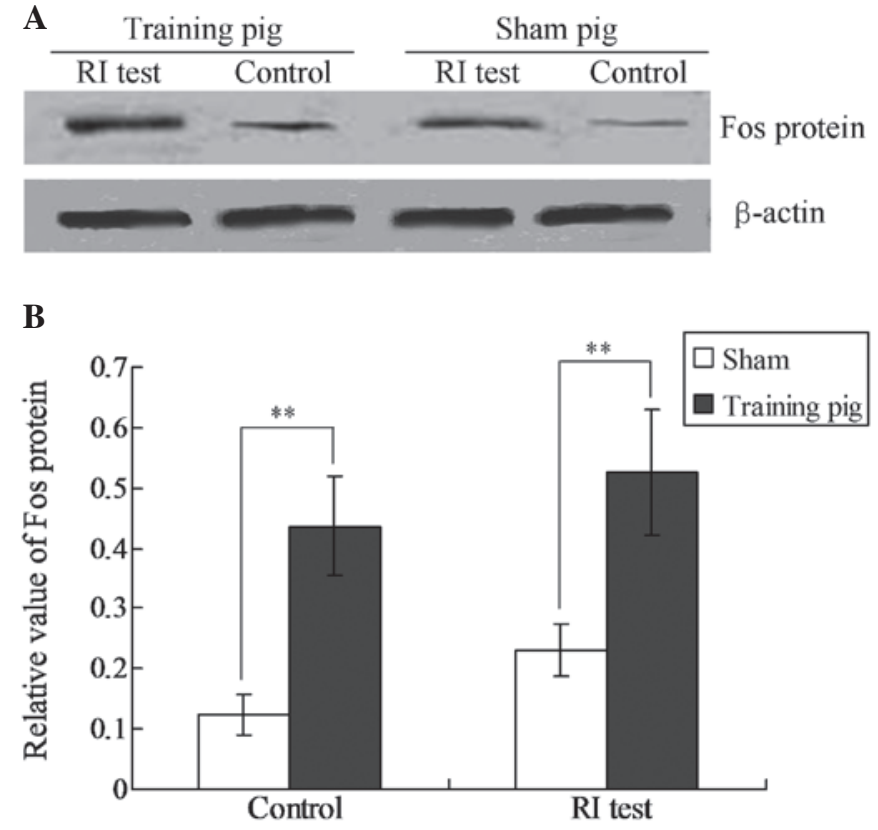

Figure 5. Fos protein expression levels in guinea pigs in the training and control groups. (A) Western blot analysis and (B) relative expression levels of Fos protein in the training and control groups. ${ }^{* *} \mathrm{P}<0.01$, vs. control guinea pigs. RI, relative-intruder.

unreported regions, including the $\mathrm{mPFC}$, to investigate different neural circuits. Notably, it was found that following CS-US paired training, microcurrent electrical stimulation of the mPFC may be used as a special CS to induce delay eyelid conditioning. However, the amplitude and acquisition speed were lower compared with those of a conditioned reflex by auditory CS.

Although the circuit involved in this CR has not been investigated experimentally, it is considered to differ from the common CR circuit. The present study hypothesized that the former is a long-loop circuit composed of multi-synapses, while the latter is a short-loop circuit with fewer synapses. This hypothesis was proposed firstly since delay eyelid conditioning has been demonstrated to be largely unaffected by forebrain lesions, engaging the cerebellum directly (15). Secondly, the output structure of delay eyelid conditioning and the nucleus of the facial nerve dose were not connected 
to the cerebellar interpositus nuclei. Finally, the mPFC is not the direct input terminal of sensory signals. Therefore, mPFC may develop an indirect association with the delay eyelid conditioning pathway via multi-synapses. Thus, the synaptic plasticity in a long-loop circuit is more difficult to construct and there is less signal attenuation in a short-loop circuit, which is consistent with the phenomena of the low amplitude and acquisition speed. However, the results demonstrated that this special CR was not stable during the training and test session. The reason for this is unclear, however, it was hypothesized that this may due to the tiny movement of electrodes; for example, the same currents form different electrode positions, which could result in significant difference (16).

This special delay eyelid conditioning appears to be more accurate and efficient in timing than auditory CR, as the distance between the CR peak and the start of the US (relative CR latency) under the stimuli of the mPFC was shorter compared with auditory stimulation. The cerebellar cortex is considered to be responsible for the accurate timing mechanism of eyelid conditioning (17); however, the signal by direct $\mathrm{CS}$ on the mPFC promotes the accuracy of CR timing. Further investigation into the underlying mechanism is required.

The delay conditioned reflex developed in the present study is the easiest eyelid conditioning to learn, while other harder eyelid conditioning, including trace eyelid conditioning and long delay eyelid conditioning, are yet to be investigated under the same conditions. In addition, the CS current used was $450 \mu \mathrm{A}$, and a smaller current may not result in the same effect. Overall, the observations of the present study may aid further investigation into the formation of eyelid conditioning and the mechanism underlying the circuit in various conditions; thus, contribute to an improved understanding of conditioned reflexes. Following the establishment of different distant cortex-induced cortical conditioning, inactivation and impairment may then be used to investigate the problem of this type of specific conditioning of neural pathways.

As aforementioned, the mechanism of eyelid conditioning has been extensively studied and the 'double parts hypothesis' has been generally acknowledged. However, in this hypothesis, the establishment of conditioning is not consistent with the 'temporal connection' hypothesis proposed by Lachnit (18). Therefore, the acquisition of eyelid conditioning does not require temporal connection to be established between the auditory cortex and body movement cortex. The afferent signals caused by an auditory stimulus and/or somatic pain stimulus reach the cerebellum concomitantly and subsequently cause synaptic plasticity changes in the cerebellar cortex and interpositus nucleus; thus, eyelid conditioning is established. Lavond and Steinmetz (19) previously reported acquisition of classical conditioning without the cerebellar cortex. Furthermore, Kelly et al (20) demonstrated that when the cerebral and cerebellar cortex of rabbits were damaged, eyelid conditioning was able to be established, although more training was required.

Therefore, as the means of support of animals has been obtained over millions of years of evolution, the conditioning reflex has simple functions, but complicated mechanisms. However, further investigation is required. In the present study, a CS was exerted on the mPFC to train guinea pigs to acquire eyelid conditioning. The results may further the understanding of different neural circuits that multiple eyelid conditioning establishment is based on. In addition, the results be used to further investigate the common mechanism of conditioning.

Eyelid conditioning is a form of associative learning and has been used extensively to study neural structures and mechanisms of learning and memory $(21,22)$. Although differing in the timing of the training stimuli, variants, including trace eyelid conditioning and delay eyelid conditioning, involve training by a CS paired with a reinforcing US to develop a CR (23). While auditory and visual stimuli are usually applied to the formation of conditioned eyelid (24), direct stimuli to the central neural pathway are also employed to induce a successful CR. Freeman and Rabinak (25) demonstrated that electrical stimulation of the pontine nuclei may be used as a $\mathrm{CS}$ in rodents, from which the similarity of the CS pathway in rats, rabbits and ferrets was identified. Furthermore, Freeman and Duffel (26) demonstrated that microstimulation of the cochlear nucleus may be used as a CS to assess developmental changes in projections to other auditory nuclei or the pontine nuclei in rats. Kalmbach et al (13) used electrical stimulation of mossy fibers as a CS to investigate the interactions between the mPFC and cerebellum. CS in the central neural pathway perform more rapid enhancement of eyelid conditioning (27). The stimulated regions in the central nerve pathway CS include brain regions, such as lateral pons, where the signal from a traditional CS passes through, and the signal transduction pathway, including parallel fibers.

However, whether eyelid conditioning is formed by a direct $\mathrm{CS}$ of the mPFC is yet to be demonstrated. A number of correlations have been reported between delay eyelid conditioning and mPFC mechanically; however, the present study, for the first time, revealed that microcurrent electrical stimulation of the mPFC may be used as a special CS to induce delay eyelid conditioning in guinea pigs. This observation may expand the current understanding of conditioned reflexes.

\section{Acknowledgements}

The study was supported by a grant from the National Natural Science Foundation of China (no. 81070875/H0902).

\section{References}

1. Vogel EH: Reinstatement of short-latency responses after asymptotic pavlovian conditioning training by the presentation of an extraneous stimulus. Biol Res 45: 61-65, 2012.

2. Chen Y, Huang F, Wang D, Weng Z and Deng Z: Upregulation of heme oxygenase-1 expression may facilitate memory and learning in mice. Exp Ther Med 5: 1491-1495, 2013.

3. Thürling M, Galuba J, Thieme A, Burciu RG, Goricke S, Beck A, Wondzinski E, Siebler M, Gerwig M, Bracha V and Timmann D: Age effects in storage and extinction of a naturally acquired conditioned eyeblink response. Neurobiol Learn Mem 109: 104-112, 2014.

4. Knowlton BJ, Lavond DG and Thompson RF: The effect of lesions of cerebellar cortex on retention of the classically conditioned eyelid response when stimulation of the lateral reticular nucleus is used as the conditioned stimulus. Behav Neural Biol 49: 293-301, 1988.

5. Green JT, Johnson TB, Goodlett CR and Steinmetz JE: Eyelid classical conditioning and interpositus nucleus activity are disrupted in adult rats exposed to ethanol as neonates. Learn Mem 9: 304-320, 2002.

6. Knowlton BJ and Thompson RF: Microinjections of local anesthetic into the pontine nuclei reduce the amplitude of the classically conditioned eyelid response. Physiol Behav 43: 855-857, 1988. 
7. Ding G, Shao J, Ding Q, Fang Z, Wu Z, Xu J and Gao P: Comparison of the characteristics of mesenchymal stem cells obtained from prostate tumors and from bone marrow cultured in conditioned medium. Exp Ther Med 4: 711-715, 2012.

8. Wu GY, Yao J, Zhang LQ, Li X, Fan ZL, Yang Y and Sui JF: Reevaluating the role of the medial prefrontal cortex in delay eyeblink conditioning. Neurobiol Learn Mem 97: 277-288, 2012

9. Yang C, Hong T, Shen J, Ding J, Dai XW, Zhou ZQ and Yang JJ: Ketamine exerts antidepressant effects and reduces IL-1 $\beta$ and IL-6 levels in rat prefrontal cortex and hippocampus. Exp Ther Med 5: 1093-1096, 2013

10. Wang Y, Liu JH, Li XH and Li L: Medial prefrontal cortex in modifying intermale aggression via oxytocin released in bed nucleus stria terminalis. J Neurol Sci Turk 29: 768-777, 2012.

11. Thompson RF and Steinmetz JE: The role of the cerebellum in classical conditioning of discrete behavioral responses. Neuroscience 162: 732-755, 2009.

12. Timmann D, Drepper J, Frings M, Maschke M, Richter S, Gerwig M and Kolb FP: The human cerebellum contributes to motor, emotional and cognitive associative learning. A review. Cortex 46: 845-857, 2010

13. Kalmbach BE, Ohyama T, Kreider JC, Riusech F and Mauk MD: Interactions between medial prefrontal cortex and cerebellum revealed by trace eyelid conditioning. Learn Mem 16: 86-95, 2009.

14. Yoshizawa K, Emoto Y, Kinoshita Y, Yuri T and Tsubura A: $\mathrm{N}$-methyl-N-nitrosourea-induced cerebellar hypoplasia in rats: Effect of arachidonic acid supplementation during the gestational, lactational and post-weaning periods. Exp Ther Med 6: 627-634, 2013.

15. McLaughlin J, Skaggs H, Churchwell J and Powell DA: Medial prefrontal cortex and pavlovian conditioning: trace versus delay conditioning. Behav Neurosci 116: 37-47, 2002.

16. Im CH, Jung HH, Choi JD, Lee SY and Jung KY: Determination of optimal electrode positions for transcranial direct current stimulation (tDCS). Phys Med Biol 53: N219-N225, 2008.
17. Attwell PJ, Ivarsson M, Millar L and Yeo CH: Cerebellar mechanisms in eyelid conditioning. Ann NY Acad Sci 978: 79-92, 2002.

18. Lachnit H: Transswitching and contextual conditioning. Relevant aspects of time. Pavlov J Biol Sci 21: 160-172, 1986.

19. Lavond DG and Steinmetz JE: Acquisition of classical conditioning without cerebellar cortex. Behav Brain Res 33: 113-164, 1989.

20. Kelly TM, Zuo CC and Bloedel JR: Classical conditioning of the eyelid reflex in the decerebrate-decerebellate rabbit. Behav Brain Res 38: 7-18, 1990.

21. Cotterill RM: Cooperation of the basal ganglia, cerebellum, sensory cerebrum and hippocampus: possible implications for cognition, consciousness, intelligence and creativity. Prog Neurobiol 64: 1-33, 2001.

22. Li B, Wang L, Liu Y, Chen Y, Zhang Z and Zhang J: Jujube promotes learning and memory in a rat model by increasing estrogen levels in the blood and nitric oxide and acetylcholine levels in the brain. Exp Ther Med 5: 1755-1759, 2013.

23. Wang H, Hu Y and Tsien JZ: Molecular and systems mechanisms of memory consolidation and storage. Prog Neurobiol 79: $123-135,2006$.

24. Steinmetz AB, Edwards CR, Steinmetz JE and Hetrick WP Comparison of auditory and visual conditioning stimuli in delay eyelid conditioning in healthy young adults. Learn Behav 37: 349-356, 2009.

25. Freeman JH and Rabinak CA: Eyelid conditioning in rats using pontine stimulation as a conditioned stimulus. Integr Physiol Behav Sci 39: 180-191, 2004.

26. Freeman JH and Duffel JW: Eyelid conditioning using cochlear nucleus stimulation as a conditioned stimulus in developing rats. Dev Psychobiol 50: 640-646, 2008.

27. Wei B, Guo Y, Zhai J, Su J, Han L, Kang C and Zhang Q: A study of the relationship between the Wnt $/ \beta$-catenin signaling pathway and the gastrointestinal development of rat embryonic and perinatal periods. Exp Ther Med 5: 1598-1602, 2013. 